\section{Penfigoide de membrana mucosa asociado a carcinoma pulmonar ¿penfigoide paraneoplásico?}

\author{
Mucous membrane penfigoid associated \\ with pulmonary carcinoma ¿paraneoplastic \\ penfigoid?
}

\section{Resumen}

El penfigoide de la membrana mucosa es una enfermedad autoinmune, que afecta a los diferentes estratos de la mucosa y la piel, y se caracteriza por lesiones vesiculo-ampollosas polimórficas. Presentando combinaciones variables de lesiones orales, oculares, cutáneas, genitales, nasofaríngeas, esofágicas y laríngeas. Su relación con las neoplasias malignas es poco conocida y sigue siendo controvertida. El presente artículo describe el manejo multidisciplinario de una paciente de 46 años de edad que desarrolló un penfigoide de membrana mucosa a nivel de la cavidad oral. Esta patología estuvo asociada a un carcinoma de pulmón, y posteriormente presentó alteraciones multiorgánicas. El tratamiento fue realizado por las especialidades de dermatología, medicina interna, cirugía general, oftalmología, estomatología, cirugía oral y maxilofacial, dicho tratamiento se llevó a cabo por etapas y tuvo una duración de 2 meses aproximadamente, logrando la resolución de las lesiones que presentaba la paciente. Consideramos que se abre una puerta para futuras investigaciones que confirman la relación entre el penfigoide de la membrana mucosa y el carcinoma de pulmón.

Palabras clave: Penfigoide benigno de la membrana mucosa; Carcinoma; Enfermedades autoinmunes (fuente: DeCS BIREME).

\begin{abstract}
Mucous membrane pemphigoid is an autoimmune disease that affects different layers from mucosa and skin, characterized by polymorphic vesicular-bullous lesions, presenting variable combinations of oral, ocular, skin, genital, nasopharyngeal, esophageal, and laryngeal lesions. Its relationship to malignant neoplasms is poorly understood and remains controversial. This article describes the multidisciplinary management of a 46-year-old patient who developed mucous membrane pemphigoid at the oral cavity level. This pathology was associated with lung carcinoma, and later presented multiorgan alterations, for which a treatment was carried out with the specialties of dermatology, internal medicine, general surgery, ophthalmology, stomatology, oral and maxillofacial surgery, said treatment was carried out in stages and lasted for approximately 2 months, achieving the resolution about lesions that patient presented. We believe that a door opens for future research confirming the relationship between mucous membrane pemphigoid and lung carcinoma.
\end{abstract}

Keywords: Pemphigoid benign mucous membrane; Carcinoma; Autoimmune diseases (source: MeSH NLM).

\section{Caso Clínico}

Samuel Urbano del Valle ${ }^{1, a, b}$, Rafael Vívero Coneo ${ }^{1, b}$ Jonathan Harris Ricardo ${ }^{1, a, c}$, Martha Carmona Lorduy ${ }^{1, a, d}$

${ }^{1}$ Universidad de Cartagena, Cartagena, Colombia.

a Especialista en Estomatología y Cirugía Oral.

${ }^{\mathrm{b}}$ Especialista en Cirugía Oral y Maxilofacial.

c Magister en Microbiología.

${ }^{d}$ Magíster en Educación.

\section{Correspondencia:}

Samuel Urbano Del Valle: surbanod@unicartagena.edu.co Barrio Zaragocilla, Campus de la Salud. Cartagena. D.T. y C. Colombia.

ORCID: 0000-0002-5773-8642

\section{Coautores:}

Rafael Vívero Coneo: rviveroc@hotmail.com ORCID: 0000-0001-9922-9937

Jonathan Harris Ricardo: j.harris.r@hotmail.com ORCID: 0000-0002-4662-0729

Martha Carmona Lorduy: mcarmonal@unicartagena.edu.co ORCID: 0000-0003-3066-2219

\section{Editora:}

Rosse Mary Falcón-Antenucci

Universidad Inca Garcilaso de la Vega, Lima, Perú.

Conflicto de intereses: los autores declaran no tener conflictos de interés.

\section{Fuente de financiamiento: ninguna}

Recibido: 02/09/20

Aceptado: 29/11/20

Publicado: 15/02/21 


\section{Introducción}

El penfigoide de membrana mucosa (PMM) o penfigoide cicatrizal es un trastorno ampollar subepitelial inflamatorio crónico autoinmune, que afecta principalmente a las membranas mucosas. Es una enfermedad rara ${ }^{1}$, en la que algunos autoanticuerpos como $\operatorname{IgG}$ en un $97 \%$, IgA $27 \%$ e IgM $12 \%$, están dirigidos contra diversos antígenos tales como bullosos 1 y 2, laminina 332, 311, colágeno tipo VII e integrina $\alpha 6 \beta 4$, localizados en zonas de membrana basal epitelial y hemidesmosomas, mientras que en el sistema del complemento C3 el 78\% está dirigido contra dichos antígenos ${ }^{2}$.

El PMM afecta con mayor frecuencia a las mujeres entre los 50-80 ańos, se manifiesta predominantemente en: mucosa oral, ocular, orofaringea, laringea y genital. La afección de piel se limita a las regiones de la cabeza, cuello y la parte superior del torso ${ }^{2}$. Las complicaciones a largo plazo del PMM incluyen sinequias, ceguera, disfagia, estenosis laríngea, estenosis anales o uretrales, entre las manifestaciones intraorales se incluyen: gingivitis descamativa, vesículas o ampollas, erosiones, pseudomembranas y úlceras ${ }^{3}$.

El análisis histopatológico muestra una división subepitelial con un infiltrado inflamatorio de eosinófilos, linfocitos y neutrófilos ${ }^{4}$. Entre las pruebas diagnósticas relevantes, la biopsia incisional permite tomar muestras de diferentes mucosas alteradas, siendo la mucosa oral el sitio de elección, por otra parte, la inmunofluorescencia directa muestra depósitos lineales de $\mathrm{IgG}$ o $\mathrm{C} 3$, y pocas veces IgA, que corroboran el diagnóstico ${ }^{4}$.

Algunos autores ${ }^{5,6}$ sugieren una posible asociación entre penfigoide y neoplasias malignas, no obstante, dicha relación sigue siendo controversial. El síndrome paraneoplásico se refiere a manifestaciones metabólicas de ciertas enfermedades malignas y que no son atribuibles a la invasión o compresión directa del tumor, así como a la diseminación distante de las células tumorales, puede afectar los sistemas endocrino, neurológico, dermatológico, reumatológico y hematológico. Se asocia con mayor frecuencia a cánceres de pulmón, especialmente de células pequeñas.

La presentación del PMM y su vínculo con cáncer de pulmón ha sido poco reportado en la literatura científica y representa un verdadero problema de salud que, de no ser tratado oportunamente, lleva a la muerte del paciente ${ }^{1,2,5}$. El objetivo es describir por medio de un caso clínico las características clínicas tanto a nivel local como sistémico del PMM, y su manejo multidisciplinario.

\section{Reporte del caso}

Anamnesis. Paciente femenina, de 46 años de edad, quien acudió al servicio de Cirugía oral y Maxilofacial del Hospital Universitario del Caribe, por presentar ampollas a nivel de cavidad oral. El acompañante de la paciente comentó que le fueron realizadas múltiples exodoncias y que la paciente después de 1 mes presentó lesiones ampollosas en la mucosa oral, ocular, vaginal e interglútea.
Examen físico general. Se observó cierre palpebral completo del ojo derecho, úlcera con secreción hemopurulenta a la palpación, en los párpados inferior y superior presentaba costra hemática, así como entropión (Figura 1), la paciente comentó pérdida de la agudeza visual. Además, presenta inicio de afección homolateral izquierda, cuya esclera se observó anictérica, con movimientos oculares conservados, párpado inferior eritematoso y epifora.

A nivel de cuello se observan movimientos de lateralidad, flexión y extensión limitados, con presencia de adenopatías a nivel submandibular y supraclavicular, a la palpación de parótida derecha, ésta se presenta tumefacta, pétrea a la palpación, de aproximadamente 5 a $6 \mathrm{~cm}$ de diámetro, asintomática, normocrómica con respecto al tejido cutáneo adyacente, de etiología desconocida y tiempo de evolución aproximadamente de 1 mes. Anasarca, tórax normoexpansible, en región interglútea presentó ampollas positivas al signo de Nikolsky y de Asboe Hanse, al igual que lesiones de tipo ampollar a nivel de región vaginal.

Examen intraoral. Se observan mucosas secas y pálidas, con ampollas en fondo de vestíbulo tanto en la arcada superior como en la arcada inferior, así como a nivel palatino y lingual, con presencia de eritema lineal en encía marginal y papilar (Figuras 2 y 3 ), en mucosa labial superior e inferior a la palpación presentó lesión nodular de aproximadamente $1 \mathrm{~cm}$ de diámetro, con secreción de material purulento. A nivel de mucosas yugal y labial se evidencia pseudomembrana que desprende al raspado, con sintomatología dolorosa a la palpación.

Examen radiográfico. En tomografía computarizada (TC) de órbita y senos paranasales, con cortes axiales y coronales, se evidenció obstrucción de los senos paranasales. En la radiografía de tórax se observó una lesión tumoral en lóbulo superior derecho, por lo que se solicita tomografía computarizada de alta resolución (TCAR) en el que se localizan lesiones tipo random o aleatorias a nivel lobulillar derecho.

Hasta este momento las características clínicas, imagenológicas y anamnesis permitieron realizar una impresión clínica de Síndrome de Steven Johnson, pénfigo vulgar o PMM.

Examen histopatológico. Se realizó biopsia incisional de lesiones a nivel palatino y vestibular, en la descripción microscópica se reportó cortes con fragmentos de una mucosa oral compuesta por un epitelio escamoso no queratinizado, con discreta acantosis y espongiosis a nivel subepitelial, se reconoce una ampolla conteniendo material hemorrágico con abundantes polimorfonucleares y depósitos de hemosiderina, el estroma subyacente presentó un infiltrado inflamatorio agudo con formación de tejido de granulación, no se reconoció malignidad en las muestras examinadas (Figura 4), hallazgos relacionados con penfigoide. Se realiza estudio de inmunofluorescencia directa siendo ésta positiva para IgG (Figura 5). 


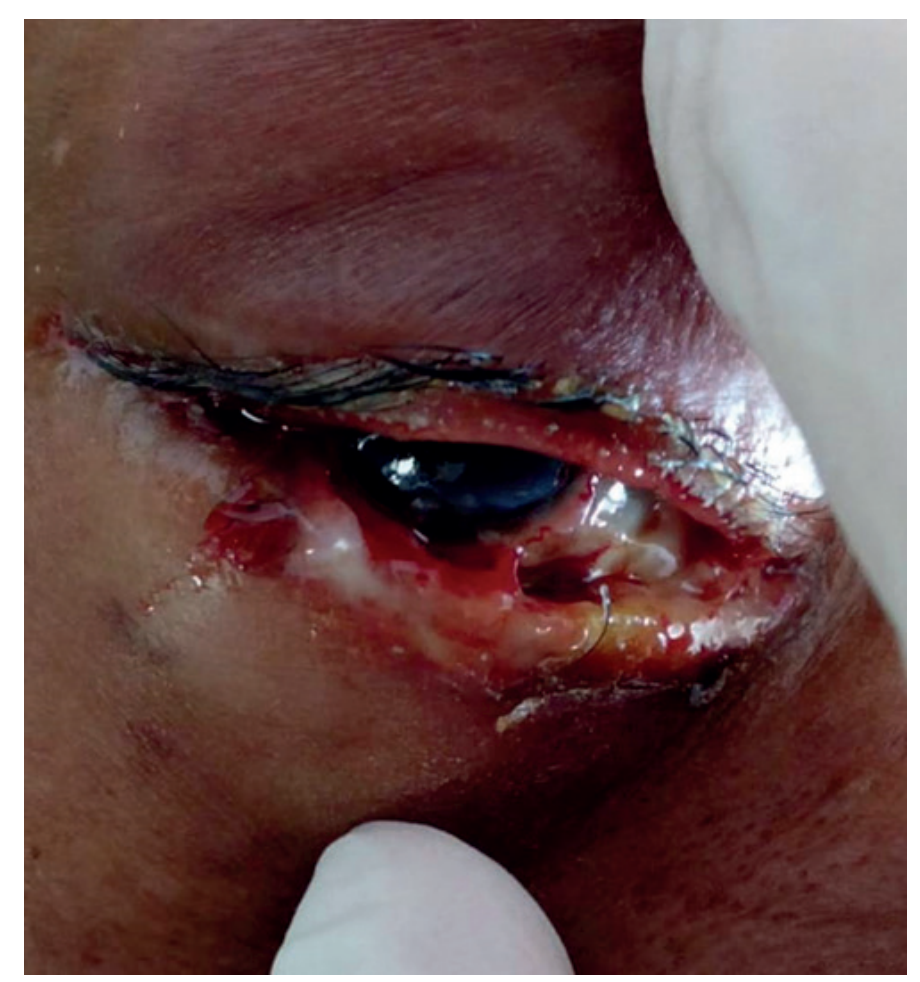

Figura 1. Ojo derecho con presencia de úlcera con secreción hemopurulenta

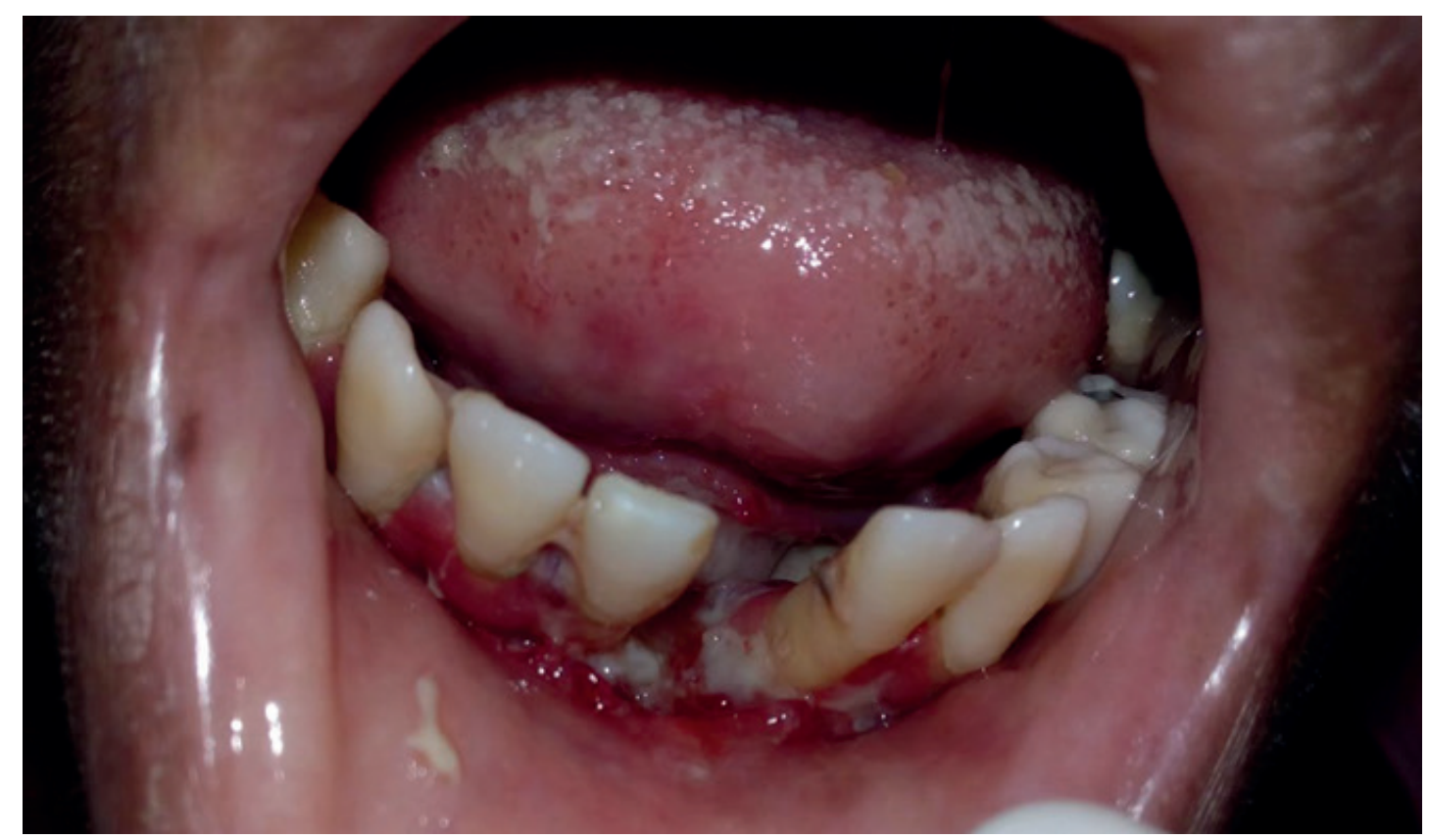

Figura 2. Eritema lineal a nivel de encía marginal y papilar de maxilar inferior

Diagnóstico. Se evaluó a la paciente en la junta médica multidisciplinar conformada por: dermatología, medicina interna, cirugía general, oftalmología, estomatología, cirugía oral y maxilofacial, en la que se decidió realizar prueba de Mantoux para descartar tuberculosis, la cual resultó negativa. Exámenes de laboratorio paraclínicos, en los cuales se evidenció disminución de los linfocitos, eritrocitos y hemoglobina, VDRL no reactivo, VIH negativo, Hepatitis $\mathrm{B}$ y $\mathrm{C}$ negativos, ionogra- ma por debajo de valores normales, BUN, Creatinina y enzimas hepáticas (GOT/GPT) dentro de parámetros normales, se realizó biopsia a nivel de lóbulo superior del pulmón derecho, con resultados histopatológicos de carcinoma pulmonar de células pequeñas. Teniendo en cuenta el examen clínico, radiográfico, histopatológico e inmunofluorescencia directa, se establece diagnóstico de PMM y CPCP. 


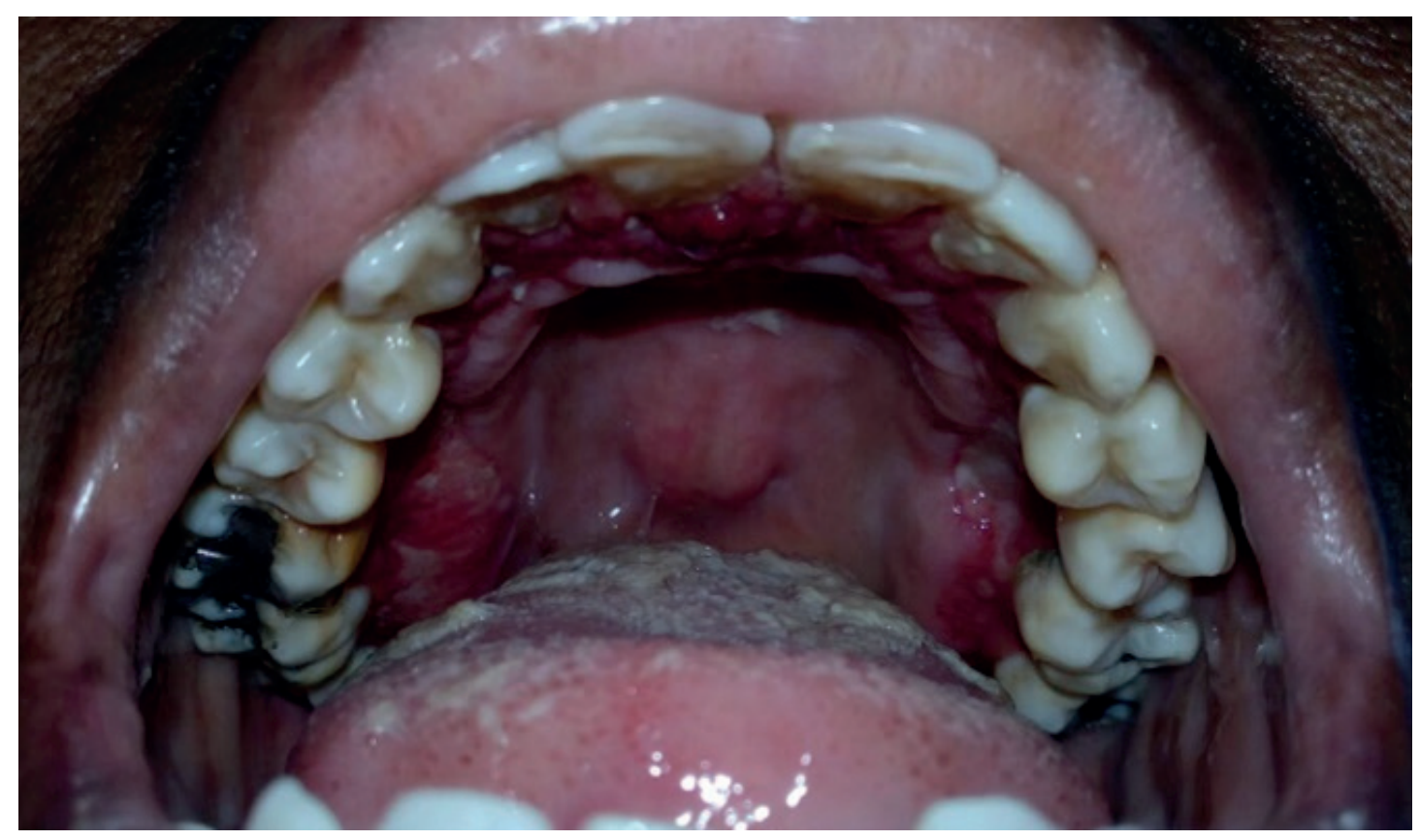

Figura 3. Eritema lineal a nivel de encía marginal y papilar de maxilar superior

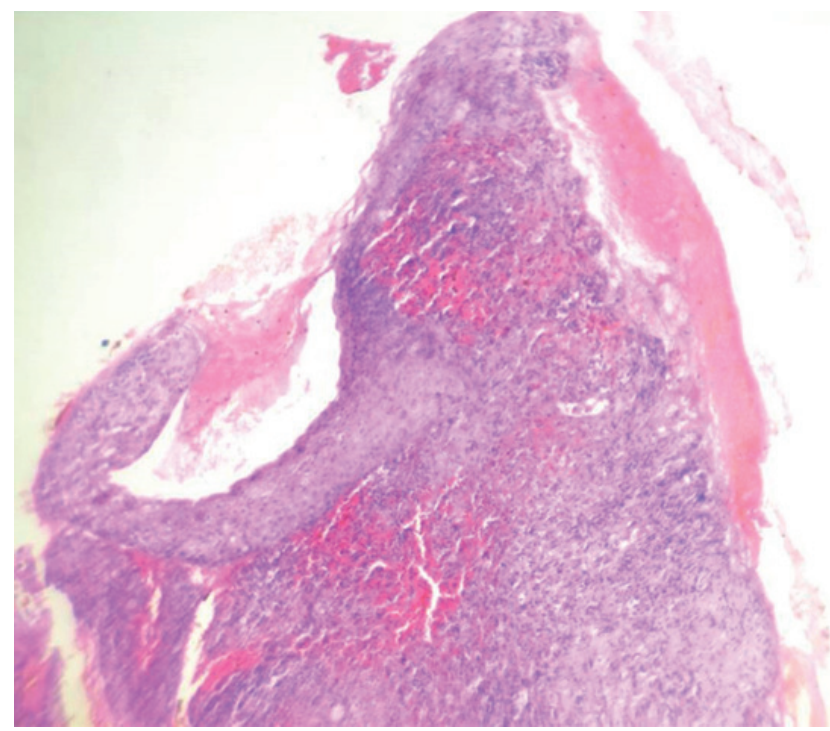

Figura 4. Biopsia incisional de lesiones de la cavidad oral

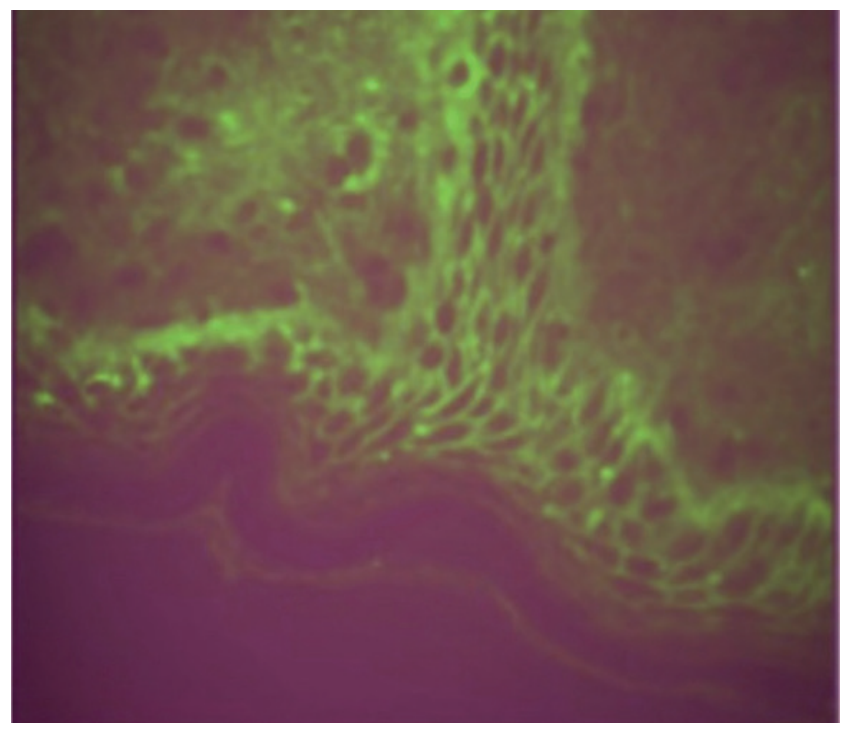

Figura 5. Estudio de inmunofluorescencia directa 
Tratamiento. La terapéutica fue secuencial, primero, el manejo de la infección fue tratada por infectología y medicina interna, con solución salina de $500 \mathrm{cc}$ más una ampolla de potasio de cloruro infundida a $60 \mathrm{cc} /$ hora, Ion sodio de $10 \mathrm{cc}$ cada 8 horas, piperacilina-tazobactam ampollas de $4.5 \mathrm{~g}$ cada 6 horas, vancomicina ampollas de $1 \mathrm{~g}$ cada 12 horas, enoxaparina ampolla de $50 \mathrm{mg}$ cada 24 horas, de manera profiláctica y tramadol ampollas de $50 \mathrm{mg}$ cada 8 horas.

Se trataron las lesiones en piel y mucosa oral, dermatología instauró manejo con Dexametasona intravenosa 4 $\mathrm{mg}$ tres veces al día durante 3 días, seguido de prednisona oral a dosis decreciente $50 \mathrm{mg} /$ día la primera semana, $30 \mathrm{mg} /$ día durante la segunda semana, $20 \mathrm{mg} /$ día en la tercera semana y $10 \mathrm{mg} /$ día la tercera semana, ungüento tópico con clobetazol, vitamina A y E cada 4 horas, aplicado a nivel vaginal y región interglútea.

Estomatología y cirugía oral, inició con eliminación de pseudoplaca en dorso de lengua, drenaje de absceso localizado a nivel de mucosa labial superior e inferior, terapia que consiste en cambiar el $\mathrm{pH}$ intraoral con bicarbonato de sodio, para evitar sobre infección de las lesiones ulcerativas, además de aplicar una base protectora de hidróxido de aluminio, hidróxido de magnesio y xilocaína en gel (orabase) en las úlceras, que ayuda en la cicatrización, de tal forma que, se cree una capa de protección de las mismas.

Se dieron recomendaciones de dieta líquida asistida, cinco días después se ordenó pautas de aplicación de Triticum vulgare, con caléndula y aloe vera en la zona, para mejorar el tiempo de cicatrización. Oftalmología describe disminución de las secreciones y lesiones eritematosas del ojo derecho, debido a la terapéutica instaurada por infectología y dermatología, se realizó examen de la agudeza visual, campos visuales, exploración de la superficie externa y musculatura del globo ocular, examen oftalmoscópico, con resultados positivos para amaurosis del ojo derecho.

En el control postoperatorio a las 3 semanas, se observó buen proceso de cicatrización, en la mucosa ocular, oral, y piel. La paciente se remitió al departamento de oncología para el manejo del CPCP. Posterior al primer ciclo de quimioterapia, el número de lesiones mucocutáneas disminuyó gradualmente y se observó una resolución completa después del segundo ciclo.

\section{Discusión}

El PMM incluye enfermedades ampollares subepiteliales autoinmunes crónicas, que afectan predominantemente a las membranas mucosas, con combinaciones variables de lesiones orales $(85 \%)$, oculares $(65 \%)$, cutáneas (25$30 \%)$, genitales $(20 \%)$, nasofaríngeas $(20-40 \%)$, esofágicas $(5-15 \%)$ y laríngeas $(5-10 \%)$, su relación con carcinomas es poco reportada y controversial ${ }^{5}$.

Lambiel et al. ${ }^{5}$ en 2017 (Reino Unido), reportaron un caso de mujer caucásica con 73 años, con PMM, la cual informó antecedentes de carcinoma de mama y un adenocarcinoma de endometrio, lo describieron como penfigoide paraneoplásico de membrana mucosa.

Gamm et al. ${ }^{7}$ en 2006 (USA), reportaron un caso inusual de una mujer con PMM y compromiso bronquial fatal, la paciente experimentó estenosis bronquial progresiva, compromiso respiratorio, que culminó con su muerte por broncoespasmo y paro cardiopulmonar ${ }^{7}$; tal y como se reporta en el presente artículo, PMM con compromiso pulmonar, particularmente $\mathrm{CPCP}$, lo que indica que el PMM podría aparecer antes que la neoplasia maligna y estar relacionado con ésta.

Trimarchi et al. ${ }^{8}$ en 2009 (Italia), afirmaron que es fundamental la combinación de estudio histopatológico e inmunofluorescencia directa, la cual muestra depósitos lineales continuos de IgG, C3 y con menos frecuencia $\operatorname{IgA}{ }^{8}$, en el actual reporte destacamos la importancia del interrogatorio y hallazgos clínicos del PMM, los cuales nos permitieron profundizar en los estudios complementando con imágenes radiográficas, histopatología, inmunofluorescencia directa con resultados positivos para IgG y CPCP.

La asociación de neoplasias malignas y PMM no es muy común, Setterfield et al. ${ }^{9}$, en el año de 1999, reportaron un caso de una mujer con penfigoide cicatrizal relacionado con carcinoma de pulmón, destacan que podría ser el primer reporte con penfigoide paraneoplásico ${ }^{9}$, luego una serie de autores ${ }^{10-13}$, los cuales, describieron casos de PMM asociado a carcinoma, como también se reporta en el presente caso; consideramos que se abre una puerta para futuras investigaciones que confirmen dicha relación y establecer la presencia de antígenos y anticuerpos relacionados, criterios de diagnóstico mayores y menores, tal cual como se presentan en el pénfigo paraneoplásico.

El manejo multidisciplinario de esta patología es importante e imprescindible, pues la presencia de múltiples lesiones a nivel sistémico, permite que, diferentes especialidades médicas deban intervenir; manejo en el que se pueda desarrollar un sistema y plan de tratamiento preciso para evaluar y medir el progreso del PMM y la neoplasia asociada ${ }^{5}$.

\section{Agradecimientos}

A la Universidad de Cartagena por permitirnos avanzar en conocimientos a través de la investigación.

\section{Referencias bibliográficas}

1. Ferreira LL, Soares GR, Valente VB, Veronese LA, Bernabé DG, Miyahara GI. Manifestação bucal de penfigóide benigno das membranas mucosas. Rev Odontol UNESP. 2014;43(especial):148. 4º Congresso Odontológico de Araçatuba 34a Jornada Acadêmica "Prof. Dr. José Eduado Rodrigues" 10 Simpósio de Pós-Graduação "Prof. Dr. Alício Rosalino Garcia" 3o Encontro de Técnicos em Laboratório "Rosimeire de Oliveira M. Gon" 60 Encontro do CAOE. 21 a 24 de maio de 2014 Faculdade de Odontologia de Araçatuba - UNESP; 2014.

2. Dharman S, Muthukrishnan A. Oral mucous membrane pemphigoid - Two case reports with varied 
clinical presentation. J Indian Soc Periodontol 2016;20(6):630-4. DOI: 10.4103/jisp.jisp_155_16.

3. Broussard KC, Leung TG, Moradi A, Thorne JE, Fine JD. Autoimmune bullous diseases with skin and eye involvement: Cicatricial pemphigoid, pemphigus vulgaris, and pemphigus paraneoplastica. Clin Dermatol. 2016;34(2):205-13. DOI: 10.1016/j.clindermatol.2015.11.006.

4. Xu H-H, Werth VP, Parisi E, Sollecito TP Mucous Membrane Pemphigoid. Dent Clin North Am. 2013;57(4):611-30. DOI: 10.1016/j.cden.2013.07.003.

5. Lambiel S, Dulguerov P, Laffitte E, Leuchter I. Paraneoplastic mucous membrane pemphigoid with ocular and laryngeal involvement. BMJ Case Rep. 2017;2017:bcr2017-220887. DOI: 10.1136/bcr-2017-220887.

6. A Das, S Das, SK Das, S Basuthakur. A case of paraneoplastic bullous pemphigoid in association with squamous cell carcinoma of lung. J Postgrad Med. 2015;61(3):1979. DOI: $10.4103 / 0022-3859.150906$.

7. Gamm DM, Harris A, Mehran RJ, Wood M, Foster CS, Mootha VV. Mucous membrane pemphigoid with fatal bronchial involvement in a seventeen-year-old girl. Cornea. 2006;25(4):474-8. Disponible en: https:// www.ophth.wisc.edu/blog/2006/05/04/mucous-membrane-pemphigoid-with-fatal-bronchial-involvement-in-a-seventeen-year-old-girl/.

8. Trimarchi M, Bellini C, Fabiano B, Gerevini S, Bussi M. Multiple mucosal involvement in cicatricial pemphigoid. Acta Otorhinolaryngol Ital. 2009;29(4):222-5. Disponible en: https://pubmed.ncbi.nlm.nih.gov/20161882/.
9. Setterfield J, Shirlaw PJ, Lazarova Z, Bryant BM, Bhogal BS, Harman K, et al. Paraneoplastic cicatricial pemphigoid. Br J Dermatol. 1999;141(1):127-31. DOI: 10.1046/j.1365-2133.1999.02933.x.

10. Ding DC, Chu TY, Hsu YH. Remission of anti-epiligrincicatricial pemphigoid after excision of cervical adenocarcinoma. J Cutan Pathol. 2014;41(8):692-3. DOI: 10.1111/cup. 12348.

11. Schmidt E, Zillikens D. Pemphigoid diseases. Lancet. 2013;381(9863):320-32. DOI: 10.1016/S01406736(12)61140-4.

12. Thorne JE, Anhalt GJ, Jabs DA. Mucous membrane pemphigoid and pseudopemphigoid. Ophthalmology. 2004;111(1):45-52. DOI: 10.1016/j.ophtha.2003.03.001.

13. Taylor J, McMillan R, Shephard M, Setterfield J, Ahmed $\mathrm{R}$, Carrozzo M, et al. World workshop on oral medicine VI: a systematic review of the treatment of mucous membrane pemphigoid. Oral Surg Oral Med Oral Pathol Oral Radiol. 2015;120(2):161-71. DOI: 10.1016/j. oooo.2015.01.024. 